\title{
A priori prediction of aggregation efficiency and rate constant for fluidized bed melt granulation
}

Kel W. Chua ${ }^{\mathrm{a}}$, Yassir T. Makkawi, ${ }^{\mathrm{b}, *}$, Michael J. Hounslow ${ }^{\mathrm{a}}$

a Particle Products Group, Department of Chemical and Biological Engineering, The University of Sheffield, Sheffield S1 3JD, United Kingdom

b Chemical Engineering and Applied Chemistry, School of Engineering and Applied Science, Aston University, Birmingham B4 7ET, United Kingdom

\section{Abstract}

This paper presents a predictive aggregation rate model for spray fluidized bed melt granulation. The aggregation rate constant was derived from probability analysis of particle-droplet contact combined with time scale analysis of droplet solidification and granule-granule collision rates. The latter was obtained using the principles of Kinetic Theory of Granular Flow (KTGF). The predicted aggregation rate constants were validated by comparison with reported experimental data for a range of binder spray rate, binder droplet size and operating granulator temperature. The developed model is particularly useful for predicting particle size distributions and growth using population balance equations (PBE).

Keyword: aggregation rate constant; fluidization; granulation; population balance equation, particle processing; particle technology.

* Corresponding author. +44 (0)121 204 3398; email: y.makkawi@aston.ac.uk 


\section{Introduction}

It is widely accepted that the engineering of particulate processes is substantially less well understood than that of fluids. Two difficulties are frequently encountered: the flow of solids is generally much more complex than that of fluids and particles can interact changing their size and shape at almost any point in a process. As a consequence fully-predictive models of fluidized bed melt granulation (FBMG) are very rare.

In FBMG molten binder is sprayed onto a bed of suspended particles. The frequent particle-particle collisions lead to bonding by solidification of the liquid bridges formed between the individual particles. Since the molten binder enters at a point and is then distributed around the bed, these processes are necessarily spatially inhomogeneous. The most widely used model for predicting the particle size distribution (PSD) and growth during granulation, namely, the Population Balance Equation (PBE), adopts a lumping approach, with part of the aggregation rate kernel derived from experiment. An active spray zone (see Fig. 1) is proposed in this paper to derive a purely theoretical model for predicting aggregation efficiency and aggregation rate constant, taking into consideration the spatially distributed processes.

\section{Theory}

The central assumption in a PBE model is that the aggregation occurs as a consequence of binary particle collisions. By analogy to second order reactions, the rate for this process is given by: 
$r_{\text {agg }}=\beta_{1,2} N_{1} N_{2}$

where $r_{a g g}$ is the rate in units of $\left[\# / \mathrm{m}^{3} \mathrm{~s}\right], \beta_{1,2}$ is the aggregation kernel for collisions between particles in size class 1 and 2 in unit of $\left[\mathrm{m}^{3} / \mathrm{s}\right], N_{1}$ and $N_{2}$ are the numbers of particles per unit volume $\left[\# / \mathrm{m}^{3}\right]$.

Hounslow (1989) has shown that $\beta_{1,2}$ for FBMG can be decomposed into a size dependence component and an aggregation rate constant $\left(\beta_{o}\right)$ such that,

$\beta_{1,2}=\beta_{0}\left(D_{1}+D_{2}\right)^{2} \sqrt{\frac{1}{D_{1}^{3}}+\frac{1}{D_{2}^{3}}}$

where $D_{1}$ and $D_{2}$ are the particle size of class 1 and 2 . The rate constant, $\beta_{o}$, can be obtained by fitting the PBE predictions of PSD to the experimental data. Combining Eqs. (1) and (2), gives the aggregation rate as follows:

$r_{\text {agg }}=\beta_{o}\left(D_{1}+D_{2}\right)^{2} \sqrt{\frac{1}{D_{1}^{3}}+\frac{1}{D_{2}^{3}}}\left(N_{1} N_{2}\right)$

In order to develop a theoretically based aggregation rate constant, Tan et al. (2004) derived an expression for $\beta_{o}$ by linking Eq. (3) with the collision rate obtained from the principles of Kinetic Theory of Granular Flow (KTGF), to give:

$\beta_{0}=\psi \cdot g_{0} \sqrt{\frac{3 \theta_{s}}{\rho_{s}}}$

where $\psi$ is a parameter representing the aggregation efficiency, $\theta_{s}$ and $g_{o}$ represent the mixture granular temperature and the radial distribution function respectively. The 
latter, $g_{o}$, is function of the solid concentration. According to Tan et al. (2004), $\theta_{s}$ and $g_{o}$ can both be obtained from suitable models, such as that based on the KTGF, while $\psi$ can be determined experimentally by fitting the measured particles size distribution into a PBE.

In a previous series of studies we discussed the time scale for four microscopic events that contribute to the overall aggregation efficiency of the process. The theoretical models predicting the time scales of (i) granule-granule collision (Chua et al, 2011a) (ii) binder droplet spreading (Chua et al, 2011b) and (iii) droplet solidification (Chua et al, 2011c) have been developed. A graphical summary of the ranges of these time scales have been presented in Chua et al. (2011a). In this paper, we make use of these models to demonstrate that such a theoretical approach can be further extended to predict the granulation efficiency $(\psi)$ and aggregation rate constant $\left(\beta_{o}\right)$, two of the most important parameters for predicting particle size growth and distribution using the population balance equation.

\subsection{Aggregation rate}

In this analysis we are interested in expressing the aggregation rate in terms of mass instead of volume, i.e. per $\mathrm{kg}$ not $\mathrm{m}^{3}$ of bed, since reference to volume of the bed has little meaning- neither the actual volume of the bed, nor that of the region where aggregation occurs. Therefore, we write the mass based rate summed over the whole fluidized bed as: 
$\bar{r}_{\text {agg }}=M_{s} \frac{d \bar{N}_{T}}{d t}=-\frac{1}{2} \int \psi r_{\text {coll }} d V$

where $M_{s}$ is the total mass in the bed, $\bar{N}_{T}$ is the number concentration per unit mass, $\psi$ is the aggregation efficiency, $\bar{r}_{\text {coll }}$ is the rate of granule-granule collision per unit volume and $V$ is the volume where aggregation takes place.

Various functions for predicting the collision rate have been reported in the literature (e.g. Kapur et al., 1969; Goldschmidt, 2001; Darelius et al., 2005). Goldschmidt (2001) has shown that, within the context of a two-fluid model, the number of collisions between particles of phases 1 and 2 per unit volume per unit time can be given by:

$r_{\text {coll }}=C_{1,2} N_{1} N_{2}$

where the collision rate constant is given by:

$$
C_{1,2}=\pi D_{1,2}^{3} g_{1,2}\left[\frac{4}{D_{1,2}}\left(\frac{\theta_{s}}{\pi} \frac{m_{1}+m_{2}}{2 m_{1} m_{2}}\right)^{1 / 2}-\frac{2}{3}\left(\nabla \bar{u}_{s}\right)\right]
$$

Following Tan et al. (2004), neglecting the divergence of the particle velocity field and assuming that all the particles are of equal density, Eqs. (6) and (7) can be combined to give:

$r_{\text {coll }}=g_{1,2} \sqrt{\frac{3 \theta_{s}}{\rho_{s}}}\left(D_{1}+D_{2}\right)^{2} \sqrt{\frac{1}{D_{1}^{3}}+\frac{1}{D_{2}^{3}}}\left(N_{1} N_{2}\right)$

Note that in Eq (8) we are assuming that $2 D_{1,2}=\left(D_{1}+D_{2}\right)$, where $D_{1,2}$ is the separation distance between two colliding particles $D_{1}$ and $D_{2}$ (centre to centre). Substituting Eq. 
(8) in Eq. (5) after replacing the volume-based number, $N$, by the mass- based number, $\bar{N}$ (i.e. $\left.N=\bar{N} \rho_{s} \varepsilon_{s}\right)$, gives:

$$
\bar{r}_{a g g}=M_{s} \frac{d \overline{N_{T}}}{d t}=-\frac{1}{2} \int \psi g_{1,2} \rho_{s}^{2}\left(\varepsilon_{s, 1} \varepsilon_{s, 2}\right) \sqrt{\frac{3 \theta_{s}}{\rho_{s}}}\left(D_{1}+D_{2}\right)^{2} \sqrt{\left(\frac{1}{D_{1}^{3}}+\frac{1}{D_{2}^{3}}\right)}\left(\bar{N}_{1} \bar{N}_{2}\right) d V
$$

\subsection{Granulation efficiency}

\subsubsection{Probability of wetting}

We start by assuming that the drops do not overlap and that the wetted area per particle is equal to a droplet cross-sectional area multiplied by a constant. It follows that for a particle with $n$ drops attached to its surface the probability that a contact point is wet is given by:

$P_{w}=n\left(\frac{k_{w} d_{o}}{D}\right)^{2}$

where $d_{o}$ and $D$ are the initial droplet and particle diameters. $k_{w}$ is a constant relating the initial droplet diameter to the final diameter after spreading. In Chua et al., 2011b, we have shown that the constant $k_{w}$ depends on the final equilibrium contact angle of the liquid binder with the spreading surface $(\omega)$ as follows:

$k_{w}=2\left[\frac{0.5 \sin ^{3} \omega}{\left(2-3 \cos \omega+\cos ^{3} \omega\right)}\right]^{1 / 3}$

If droplets collide with granules in an uncorrelated fashion, then over a time period $t$, the probability that the number of droplets on a granule's surface is $n$, will be given by a Poisson distribution as follows: 
$P_{n}(t)=\frac{\left(t / \tau_{D-G}\right)^{n}}{n !} e^{-t / \tau_{D-G}}$

where $\tau_{D-G}$ is the droplet-granule collision time discussed below. It is not clear how many active droplets will be attached to the particle when it contact, however, we know that it is dependent on the duration of the time allowed for a particle to collect droplets. If we assume the maximum of this duration is equal to the droplet solidification time $\tau_{s}$ then summing Eq. (12) over all values of $n$ and multiplying by Eq. (10) gives:

$$
P_{w}=n\left(\frac{k_{w} d_{o}}{D}\right)^{2} \sum_{n=0}^{\infty} P_{n}(t)=\frac{\tau_{s}}{\tau_{D-G}}\left(\frac{k_{w} d_{o}}{D}\right)^{2}
$$

Both of the time scales, $\tau_{s}$ and $\tau_{D-G}$ can be computed theoretically. In Chua et al. (2011c), we have shown that $\tau_{s}$ can be given by:

$\tau_{s}=2.03\left[\frac{d_{o} \rho_{l} \lambda}{k_{w}^{2} \alpha^{0.5} \rho_{s} C_{p s}\left(T_{m}-T_{s}\right)}\right]^{2}$

where $\rho_{l}, \alpha$ and $\lambda$ are the binder density, thermal diffusivity and latent heat of fusion respectively, $\rho_{s}$ and $C_{p s}$ are the particle density and specific heat capacity respectively, $T_{m}$ and $T_{s}$ are the melting temperature of the binder and temperature of the particle respectively. We also have shown in Chua et al. (2011a) that the droplet-granule collision time, $\tau_{D-G}$, can be given by the following Ballistic model (high-velocity droplets) (assuming a spherical sector spray zone):

$\tau_{D-G}=\frac{4 \pi(1-\cos \phi) r^{2} d_{o}^{3} \rho_{L}}{3\left(D+d_{o}\right)^{2} \dot{M}_{D}} \exp \left(\frac{3\left(D+d_{o}\right)^{2}}{2 D^{3}} \varepsilon_{s} r\right)$ 
Substituting Eqs. (14) and (15) in Eq. (13) gives the probability of wet surface contact as follows:

$$
P_{w}=\frac{3 d_{o} \rho_{L} \lambda^{2} \dot{M}_{D}}{k_{w}^{2} \alpha \rho_{s}^{2} C_{c p}^{2}\left(T_{m}-T_{s}\right)^{2} 2 \pi(1-\cos \phi) r^{2}}\left(\frac{D+d_{o}}{D}\right)^{2} \exp \left[-\frac{3\left(D+d_{o}\right)^{2}}{2 D^{3}} \varepsilon_{s} r\right]
$$

Now, making due allowance that this probability must be limited to 1 , we write:

$$
P_{w}=M I N\left[1,\left(\frac{k_{w} d_{o}}{D}\right)^{2} \frac{\tau_{s}}{\tau_{D-G}}\right]
$$

\subsubsection{Aggregation efficiency}

We assume that the efficiency, $\psi$, can be expressed in term of the probability of wet surfaces contact, $P_{w}$, such that,

$\psi=1-\left(1-P_{w}\right)^{2}$

The use of Eq. (18) in the integral form of the aggregation rate given earlier in Eq. (9) is difficult; therefore, we make a simplification, without jeopardizing the solution accuracy, by assuming that Eq. (18) can be approximated by:

$\psi \approx 2 P_{w}$

which then, after substituting $P_{w}$ given in Eq. (16), reduces to the final efficiency form,

$\psi \approx \frac{6 d_{o} \rho_{L} \lambda^{2} \dot{M}_{D}}{k_{w}^{2} \alpha \rho_{s}^{2} C_{c p}^{2}\left(T_{m}-T_{s}\right)^{2} 2 \pi(1-\cos \phi) r^{2}}\left(\frac{D+d_{o}}{D}\right)^{2} \exp \left[-\frac{3\left(D+d_{o}\right)^{2}}{2 D^{3}} \varepsilon_{s} r\right]$ 
Similar to the allowance used in Eq. (17), we limit the maximum aggregation efficiency to 1 . A comparison of the aggregation efficiency values given by Eqs. (18) and (19) is shown in Fig. 2. Both expressions appear to exhibit the same behaviour with negligible numerical differences.

\subsection{Average aggregation rate constant}

In section 2.1 we have shown how a mass based aggregation rate [Eq. (9)] can be obtained by making use of collision rates obtained from the principles of KTGF. Now, comparing Eq. (3) with Eq. (9) lead to the following mass-based aggregation rate constant:

$\bar{\beta}_{o}=\frac{\sqrt{3} \rho_{s}^{3 / 2}}{2 M_{s}} \int \psi g_{1,2} \sqrt{\theta_{s}}\left(\varepsilon_{s, 1} \varepsilon_{s, 2}\right) d V$

For mono-disperse granules, or where volume fraction does not vary with size, and defining the spray zone, of volume $V_{s z}$, as the region in which binder is available, Eq. (21) takes the form:

$\bar{\beta}_{o}=\frac{\sqrt{3} \rho_{s}^{3 / 2}}{2 M_{s}} \int_{s z} g_{o} \varepsilon_{s}^{2} \sqrt{\theta_{s}} \psi d V_{s z}$

For a symmetrical spray zone in the form a spherical sector of half angle $\phi$ (see Fig. 1), with $d V_{s z} \approx 2 \pi(1-\cos \phi) r^{2} d r$, the aggregation rate constant as a function of distance, $r$, from the spray zone tip is given by: 
$\bar{\beta}_{o}=\frac{\pi \sqrt{3}(1-\cos \phi) \rho_{s}^{3 / 2}}{M_{s}} \int_{0}^{\infty} g_{o} \varepsilon_{s}^{2} \sqrt{\theta_{s}} \psi r^{2} d r$

In order to calculate the rate constant, the integral part in Eq. (23) must be determined by, for example, evaluating the areas under the curves in Fig. 3. Numerical evaluation of those integrals gives, for particle diameters of $175 \mu \mathrm{m}, 200 \mu \mathrm{m}$ and $300 \mu \mathrm{m}$ values for $\bar{\beta}_{o}$ of $2.78 \times 10^{-8} \mathrm{kgm}^{-0.5} \mathrm{~s}^{-1}, 2.77 \times 10^{-8} \mathrm{kgm}^{-0.5} \mathrm{~s}^{-1}$ and $2.84 \times 10^{-8} \mathrm{kgm}^{-0.5} \mathrm{~s}^{-1}$ respectively. It is clear that $\bar{\beta}_{o}$ is nearly constant for the three different sizes considered, thus in good agreement with the experimental observations of Tan et al. (2006).

If for simplification we assume that the spray zone is hydrodynamically uniform, with $\varepsilon_{s}$ and $\theta_{s}$ independent of $r$, then Eq. (23) can be written to give an average aggregation rate constant as follows:

$\beta_{o}=\frac{\pi \sqrt{3}(1-\cos \phi) \rho_{s}^{3 / 2}}{M_{s}} g_{o} \varepsilon_{s}^{2} \sqrt{\theta_{s}} \int_{0}^{\infty} \psi r^{2} d r$

In this equation, the lumped quantity $\varepsilon_{s}^{2} \sqrt{\theta}$ can be resolved from Computational Fluid Dynamic (CFD) simulation (see Chua et al., 2011) and $g_{o}$ is function of $\varepsilon_{s}$. The quantity under the integral can be determined by making use of the approximate aggregation efficiency given in Eq. (20), such that:

$$
\int_{0}^{\infty} \psi r^{2} d r \approx \frac{2 d_{o} \rho_{l} \lambda^{2} \dot{M}_{D} D}{k_{w}^{2} \alpha \rho_{s s}^{2} C_{p s}^{2}\left(T_{m}-T_{s}\right)^{2} \pi(1-\cos \phi) \varepsilon_{s}}
$$


Finally, substituting Eq. (25) in Eq. (24) gives the following approximate average aggregation rate constant:

$$
\bar{\beta}_{o} \approx 3.5 g_{o} \varepsilon_{s} \sqrt{\theta_{s}} \frac{\dot{M}_{D}}{M_{s}} \frac{D d_{o} \rho_{l} \lambda^{2}}{k_{w}^{2} \alpha \rho_{s}^{0.5} C_{c p}^{2}\left(T_{w}-T_{s}\right)^{2}}
$$

In practice, this equation allows for the determination of the average aggregation rate constant once the granulator operating conditions are known. The hydrodynamic parameters, $\varepsilon_{s}$ and $\theta_{s}$ can be obtained, to a good degree of accuracy, using available CFD techniques as we have shown in Chua et al. (2011a).

\subsection{Probability of liquid bridge rupture}

Previous experimental investigations by Tan et al. (2006) have shown that the bed temperature has two competing effects on the aggregation rate constant depending on the bed temperature: (i) enhanced binder spreading at low bed temperature, thus increasing the wet fractional coverage of the particle, or in other words, increasing the probability of granule-granule contacts at the wet surface (ii) granules breakage at high bed temperature close to the binder melting point, which is believed to be due to increasing probability of liquid bridge rupture with decreasing the binder viscosity. While the above proposed formulations indeed takes into consideration the effect of bed temperature on the aggregation rate constant through the binder solidification term, it lacks the appropriate formulation to account for this effect on the probability of liquid bridge rupture as the bed temperature increases (i.e. due to reduced binder viscosity). 
For this purpose, it is proposed to quantify the probability of liquid bridge survival at high temperature by using Ennis (1991) force balance between binder viscosity and particle inertia expressed in terms of the Stokes number. The Stokes number is defined in terms of the ratio of initial particle kinetic energy to the energy dissipation at collision as follows: $S t=\frac{8 m u_{o}}{3 \pi \mu D^{2}}$

where $u_{o}$ is the inter-particle approach velocity (collision velocity) and $\mu$ is the binder viscosity, given as function of temperature as follows (Chua et al. 2001b):

$\mu=\exp \left[-9.207 \ln \left(\frac{T}{T_{o}}\right)-0.5274\right]$

where $T$ is the temperature in Kelvin and $T_{o}=273 \mathrm{~K}$.

From the principles of kinetic theory of granular flow, $u_{o}$ can be obtained in terms of the granulation temperature, $\theta$, such that,

$u_{o}=\frac{3}{2} \sqrt{\pi \theta}$

Thielmann et al. (2007) identified a critical stokes number, above which the liquid bridge rupture becomes effective, as follows:

$$
S t^{*}=\left(1+\frac{1}{e}\right) \ln \left(\frac{h}{h_{a}}\right)
$$

where $e$ is the particle-particle restitution coefficient (taken here as 0.95), $h$ and $h_{a}$ are the thickness of the binder layer and the characteristic measure of the particle surface 
asperity respectively. Based on experimental evaluations for fine-grained particles, Krupp (1976) suggested a value of $0.1 \mu \mathrm{m}$ for the surface asperity. Thielmann et al. (2007) suggested a value of $2.5 \mu \mathrm{m}$ and Makkawi (2004) identified this to fall within the range of $0.08-6 \mu \mathrm{m}$ for small glass beads in the range of $90-1850 \mu \mathrm{m}$. For the particle size and type considered in this study, an average value of $h_{a}=5 \mu \mathrm{m}$ is used. The binder thickness over the particle surface is estimated using geometrical analysis of binder base area and the contact angle, such that,

$h=\frac{d_{f}}{2}\left(\frac{1-\cos \omega}{\sin \omega}\right)$

where $\omega$ is the particle-liquid binder contact angle and $d_{f}$ is the diameter of the final spreaded liquid droplet on the particle surface, given by $d_{f}=k_{w} d_{o}$ (Chua et al., 2011b). Finally, and following a similar approach suggested by Thielmann et al. (2007), the probability of liquid bridge survival, $P_{s}$ is described mathematically as follows,

$$
P_{s}= \begin{cases}1 & S t \leq S t^{*} \\ e^{v\left(s t^{*}-s t\right)} & S t>S t^{*}\end{cases}
$$

where $v$ is an adjustable parameter introduced due to uncertainly in the value of $S t^{*}$. According to Eq. 32, particles colliding with $S t \leq S t^{*}$ are of low inertia that considerably dissipates upon collision; hence coalescence and subsequent liquid bridge solidification occur. Particles colliding with $S t>S t^{*}$ are of high inertia that give rise to rebound after collision and hence, result in exponential decay of liquid bridge survival probability. The probability of survival obtained by using Eq. (32) is demonstrated in Fig. 4 for a selected range of parameters. Note that the probability of liquid bridge survival exponentially 
decays after reaching a given bed temperature and then gradually approaches zero as the temperature gets closer to the binder melting point of $44{ }^{\circ} \mathrm{C}$.

Now, incorporating the proposed probability of liquid bridge survival in the aggregation rate constant, given earlier in Eq. (27), we obtain the following modified version:

$$
\bar{\beta}_{o} \approx 3.5 g_{o} \varepsilon_{s} \sqrt{\theta_{s}} \frac{\dot{M}_{D}}{M_{s}} \frac{D d_{o} \rho_{l} \lambda^{2}}{k_{w}^{2} \alpha \rho_{s}^{0.5} C_{c p}^{2}\left(T_{w}-T_{s}\right)^{2}}\left[P_{s}\right]
$$

\section{Parametric analysis and model validation}

We present in this section comparison of the predicted aggregation rate constant with experimental data for a range of fluidized bed temperature, binder droplet sizes and spray rates. These three parameters play a major role in defining the fluidized bed granulator performance.

\subsection{Binder spray rate}

In the previous sections, we have shown, through theoretical modelling, that the aggregation rate constant is directly proportional to the binder spray rate [see Eq. (26)]. At the single particle level, the increase in binder spray rate will result in increasing the wet particle area, which upon collision may lead to successful agglomeration. Fig. 5 shows a comparison between the calculated aggregation rate constant and experimental data obtained from Tan et al. (2006). The results confirm reasonable agreement between our model prediction and the reported experiment data. Most 
important, this figure demonstrates the critical sensitivity of the fluidized bed granulator performance with respect to the binder spray rate.

\subsection{Binder droplet size}

In fluidized bed granulation, the binder droplet size is controlled by the atomizing air pressure. Fig. 6 shows the predicted aggregation rate constant in comparison with experimental data for an atomizing air pressure of 1 to 2 bars, which corresponds to a droplet size in the range of $18.5-39.5 \mu \mathrm{m}$. It is clear that the predictions follow the same experimental trend and are quantitatively valid, especially at high binder droplet size. The results also indicate that, within the range considered, the aggregation rate constant increases with increasing binder droplet size. This is mainly due to the enhanced particles wetting and to the reduction in the binder solidification time, both are highly desirable features for successful aggregation (see Chua et al., 2011b,c).

\subsection{Fluidized bed temperature}

Fig. 7 shows the predicted aggregation rate constants, using Eq. (26) and its modified version Eq. (33), in comparison with the experimental data of Tan et al. (2006) for different bed temperatures. The aggregation rate constant given by Eq. (27) shows critical deviations as the bed temperature increases $\left(T>34{ }^{\circ} \mathrm{C}\right)$. This is due to a recognized limitation of Eq. (26) in predicting the effect of liquid bridge rupture, which in this case is due to the decreased in binder viscosity. The modified equation [Eq. (33)], in which such an effect is incorporated through the probability of liquid bridge survival term, appear to show a relatively better match with the experimental data as indicated by the 
dip in aggregation rate constant at high bed temperatures. However, it must be noted that such a corrective approach is critically sensitive to the parameters used and requires careful setting of the adjustable parameter $v$, which in this case is equal to 30 .

An alternative approach to interpreting the data in Fig. 7 is to use the ratio of the predicted to the measurement aggregation rate constants $\left(\bar{\beta}_{\text {o-predicted }} / \bar{\beta}_{\text {o-measured }}\right)$ for approximate quantification of the probability of particle breakage, or what we referred to by liquid bridge survival. This will not be discussed in this paper and it is open for future investigations and debate.

\section{Conclusion}

We have shown that the aggregation rate for spray fluidized bed granulation can be derived by combining a probability analysis of particle-droplet contact with time scale analysis of granule-granule collisions and droplet solidification rates. This is an important step for future development of new generations of theoretical models for the prediction of particle size distribution and size growth in a fluidized bed melt granulator.

The model given by Eq. (26) provided quantitatively valid data and successfully predicted the experimentally observed linear relationship between the aggregation rate constant and the binder droplet size and injection rate. However, Eq. (26) is only accurate for low bed temperature, well below the binder melting point, or when the effect of liquid bridge rupture is negligible. To overcome this limitation, we proposed a modified aggregation rate constant [(Eq. (33)] by introducing liquid bridge survival probability term. 
This appears to produce qualitatively improved predictions that are reasonably matching the experimentally observed behaviour at high operating temperature. However, Eq. (33) must be treated carefully as it is critically sensitive to the various parameters used and requires setting of an adjustable parameter. 


\section{Notation}

$C_{1,2}$

$C_{p s}$

e

$d_{o}, d_{f}$

$D_{1,2}$

D

$k_{w}$

$\dot{M}_{D}$

$M_{s}$

$m$

$n$

$N$

$\bar{N}$

$\bar{N}_{T}$

$P_{W}$

$P_{n}$

$P_{s}$

$r$

$r_{a g g}, \bar{r}_{a g g}$

$r_{\text {coll }}$ collision rate constant $\left(\mathrm{m}^{3} \mathrm{~s}^{-1}\right)$

specific heat capacity of solid $\left(\mathrm{J} \mathrm{kg}^{-1}{ }^{\circ} \mathrm{C}^{-1}\right)$

particle-particle restitution coefficient (-)

spray droplet diameter before and after spreading respectively (m)

Inter-particle distance between two colliding particles (m)

representative size of granule through granulation process $(\mathrm{m})$

ratio of final droplet cross-sectional area to initial droplet area (-)

droplet injection rate $\left(\mathrm{g} \mathrm{min}^{-1}\right)$

mass of solid in granulator $(\mathrm{kg})$

mass of a single particle $(\mathrm{kg})$

number of binder droplets attached to a particle (-)

number concentration of particle per unit volume $\left(\# \mathrm{~m}^{-3}\right)$

number concentration of particle per unit mass $\left(\# \mathrm{~kg}^{-1}\right)$

total number of particles in the fluidized bed (-)

probability of wet contacts (-)

probability of $n$ number of liquid drops on a particle (-)

probability of liquid bridge survival (-)

distance from the spray nozzle tip $(\mathrm{m})$

volume and mass based aggregation rates respectively $\left(\# \mathrm{~m}^{-3} \mathrm{~s}^{-1}, \# \mathrm{~kg}^{-1} \mathrm{~s}^{-1}\right.$ )

rate of granule-granule collision $\left(\# \mathrm{~m}^{-3} \mathrm{~s}^{-1}\right)$ 


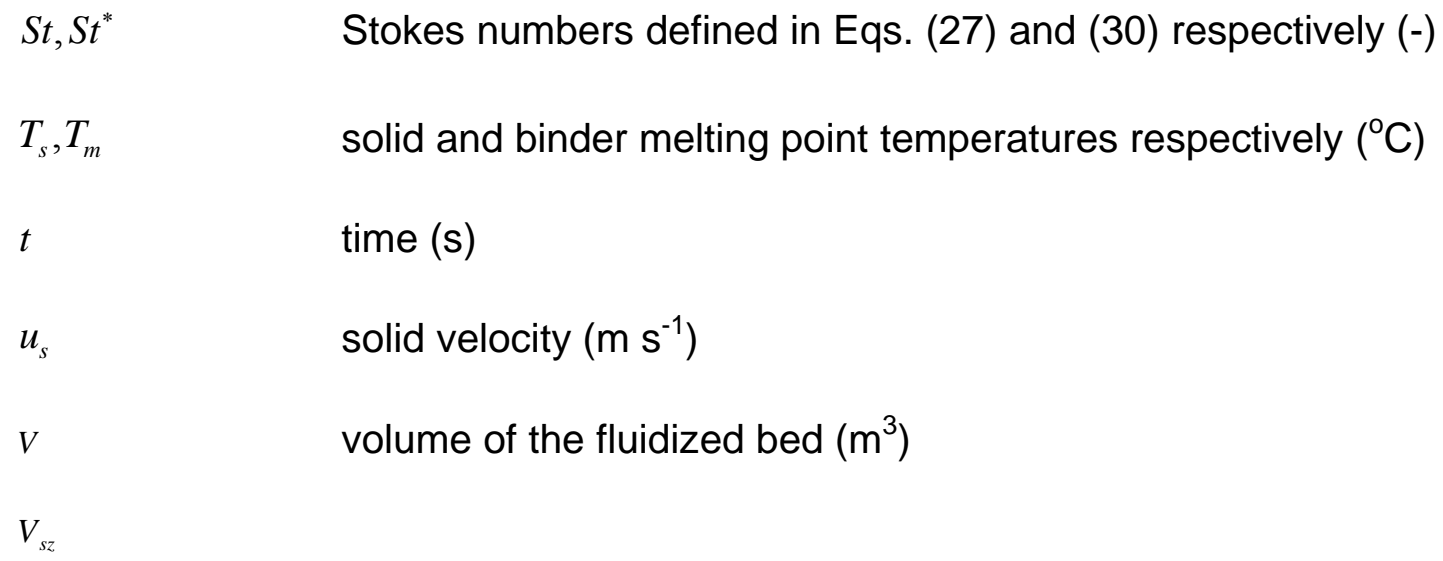




$$
\begin{array}{ll}
\mu & \text { Viscosity of the binder }\left(\mathrm{kg} \mathrm{m}^{-1} \mathrm{~s}^{-1}\right) \\
\eta & \text { particle-particle restitution coefficient (-) }
\end{array}
$$

\section{References}

Chua, K. W., Makkawi, Y. M., Hounslow, M. J., 2011a. Time scale analysis for fluidized bed melt granulation- I: granule-granule and granule-droplet collision rates. Chemical Engineering Science, Volume 66, Issue 3, 318-326.

Chua, K. W., Makkawi, Y. M., Hounslow, M. J., 2011b. Time scale analysis for fluidized bed melt granulation- II: binder spreading rate. Chemical Engineering Science, Volume 66 , Issue 3, 327-335.

Chua, K. W., Makkawi, Y. M., Hounslow, M. J., 2011c. Time scale analysis for fluidized bed melt granulation- III: binder solidification rate. Chemical Engineering Science, Volume 66, Issue 3, 336-341.

Darelius, A., Rasmuson, A., Bjorn, I. N., Folestad, S., 2005. High shear wet granulation Modeling- a mechanistic approach using population balances. Powder Technology 160, 209-218.

Ennis, B. J., Tardos, G., Pfeffer, R., 1991. A microlevel-based characterization of granulation phenomena, Powder Technology. 56, 257-272. 
GoldSchmidt, M. J. V., 2001. Hydrodynamic Modelling of Fluidized Bed Granulation. PhD thesis, Twente University.

Hounslow, M. J., Ryall R. L., 1988. A discretized population balance for nucleation, growth and aggregation. AIChE Journal 34 (11), 1821-1832.

Kapur, P. C., Fuerstenau, D. W., 1969. Coalescence model for granulation, Industrial and Engineering Chemistry, Process Design Devices, 56-62.

Krupp, H. (1967). Particle adhesion. Advances in Colloid and Interface Science, 1, 111239.

Makkawi, Y. M., 2004. Investigation of dry and semi-wet fluidized bed hydrodynamics utilizing rwin-plane electrical capacitance tomography. PhD thesis, Heriot-Watt University, Edinburgh, UK.

Tan, H. S., Goldschmidt, M. J. V., Boerefijn, R., Hounslow, M. J., Salman, A. D., Kuipers, J. A. M., 2004. Building population balance for fluidized bed granulation: lessons from kinetic theory of granular flow. Powder Technology 142, 103-109.

Tan, H. S., Salman, A. D., Hounslow, M. J., 2006. Kinetics of fluidized bed melt granulation-II: Modelling the net rate of growth. Chemical Engineering Science, 61, 3930-3941. 
Thielmann, F., Naderi, M., Ansari, M., Stepanek, F., 2007. The effect of primary particle surface energy on agglomeration rate in fluidised bed wet granulation. Powder Technology 181, 160-168. 


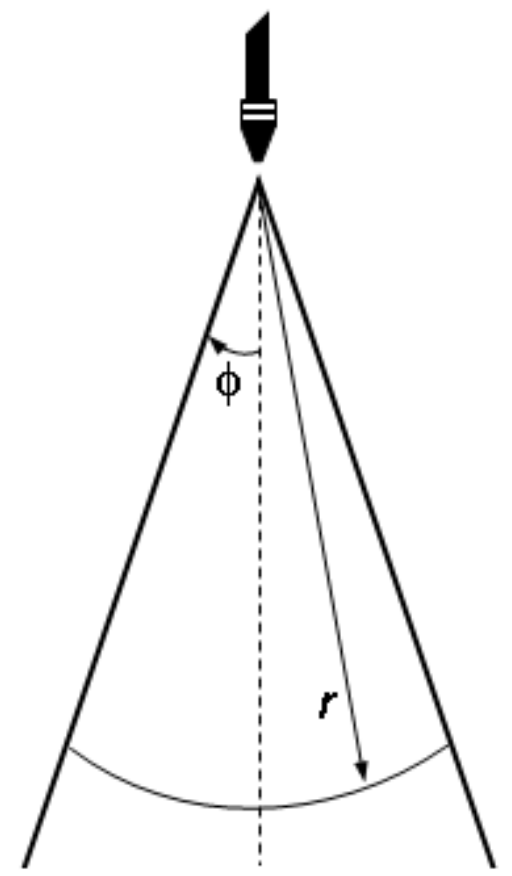

Fig. 1. Schematic representation of spray zone in a fluidized bed spray melt granulator 


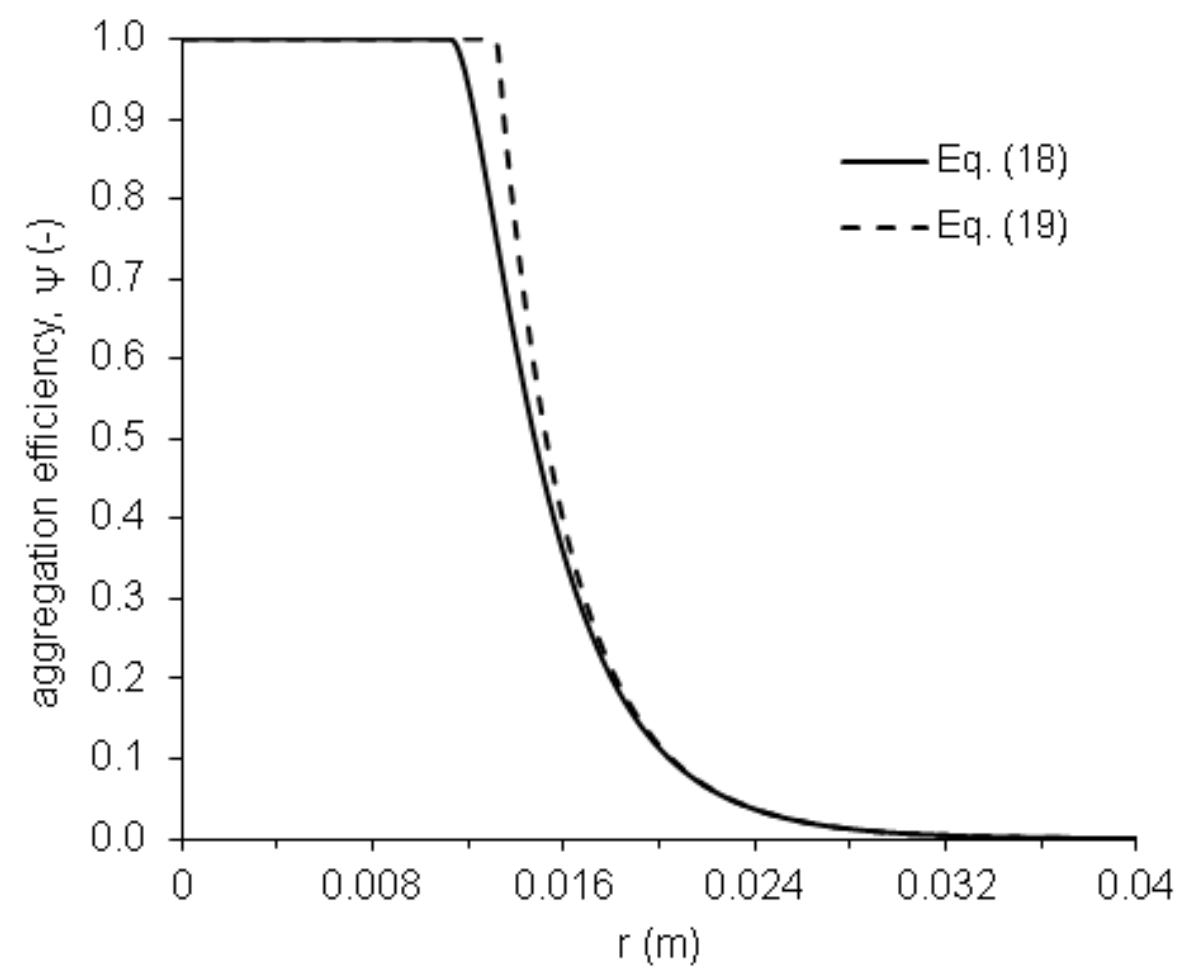

Fig. 2. Aggregation efficiency as function of the distance from the spray nozzle estimated using Eqs. (18) and (19). The parameters used in the equations are: $d_{o}=40$ $\mu \mathrm{m}, \rho_{l}=1093 \mathrm{kgm}^{-3}, \rho_{\mathrm{s}}=2500 \mathrm{kgm}^{-3}, \dot{M}_{D}=8 \mathrm{gmin}^{-1}, \lambda=155 \mathrm{kJkg}^{-1}, \alpha=5.7 \times 10^{-7} \mathrm{~m}^{2} \mathrm{~s}^{-1}$, $C_{p}=0.84 \mathrm{kJkg}^{-1} \mathrm{~K}^{-1}, T_{m}=44{ }^{\circ} \mathrm{C}, T_{s}=34{ }^{\circ} \mathrm{C}, \phi=22.5^{\circ}, \omega=35.0^{\circ}$. 


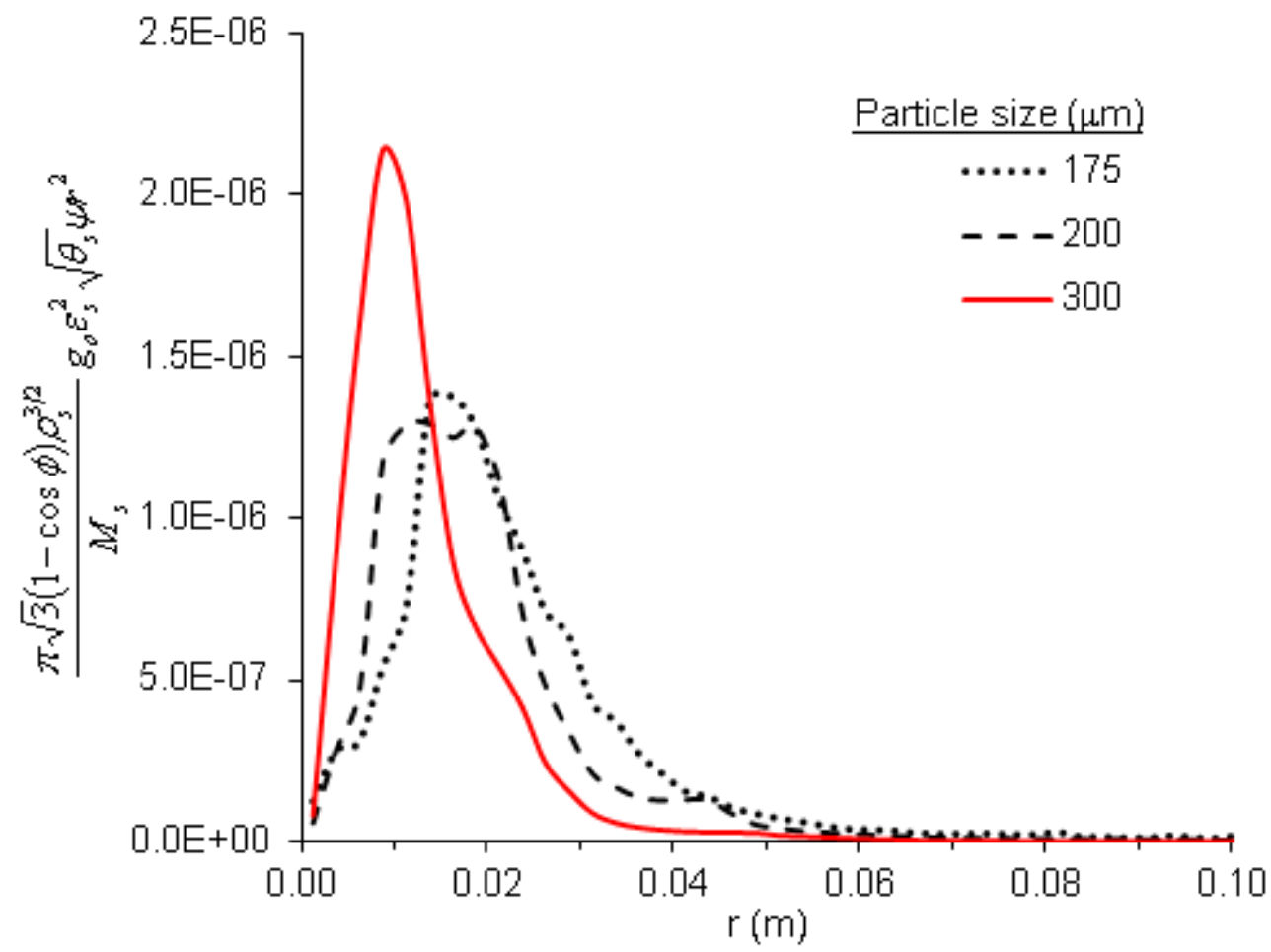

Fig. 3. Graphical determination of the average aggregation rate constant, $\bar{\beta}_{o}$, for three different particle sizes using Eq. (23). $\bar{\beta}_{o}$ is given by the area under the curve. 


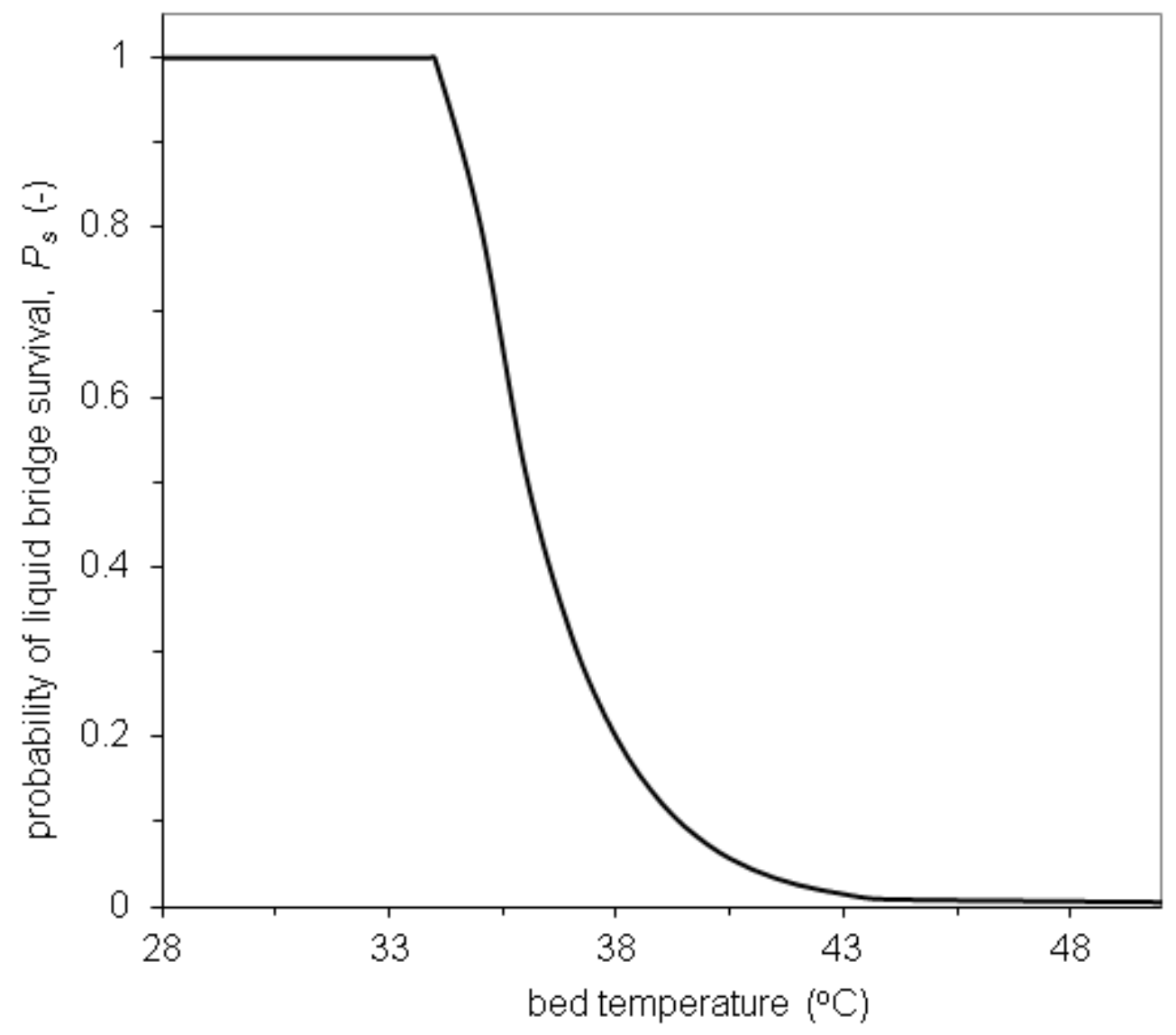

Fig. 4. Probability of liquid bridge survival as function of the fluidized bed operating temperature estimated using Eq. (32). The parameters used are: liquid binder PEG1500 with properties, $d_{o}=20 \mu \mathrm{m}, \mu=$ function of temperature (see Chua et al. 2011b), $\phi=$ $22.5^{\circ}, \theta=0.012 \mathrm{~m}^{2} \mathrm{~s}^{-2}, \vartheta=35.0^{\circ}, \eta=0.95, D=300 \mu \mathrm{m}, h_{o}=5 \mu \mathrm{m}, 2500 \mathrm{kgm}^{-3}, \rho_{\mathrm{s}}=$ $2500 \mathrm{kgm}^{-3}, n=30$. 


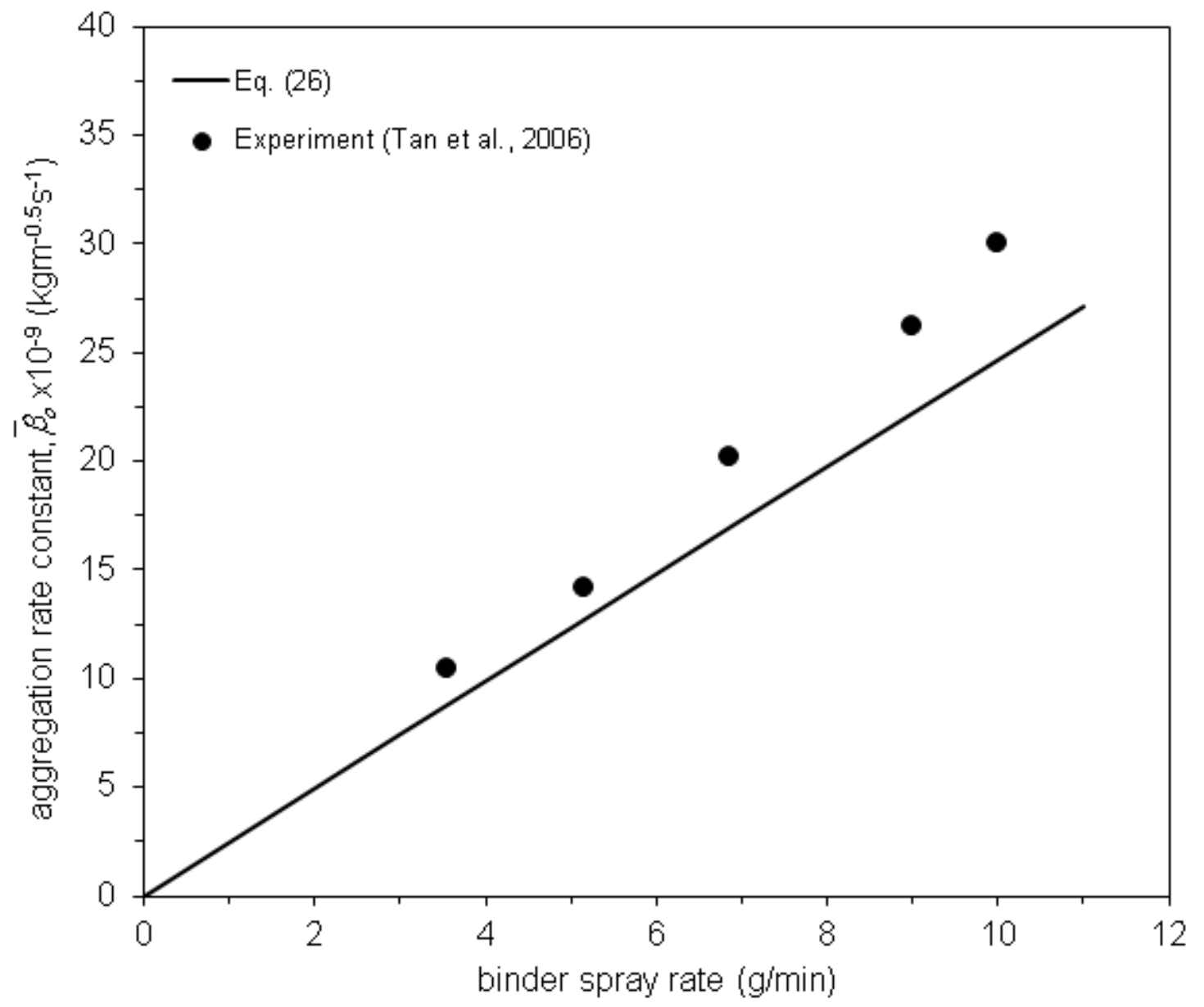

Fig. 5. Comparison of experimental and predicted aggregation rate constant using Eq. (27). The experiment of Tan et al. (2006) was carried out with glass beads and PEG1500 as a binder, spray nozzle pressure of 1.5 bar, fluidizing air velocity of $0.97 \mathrm{~ms}^{-1}$ and a bed temperature of $32{ }^{\circ} \mathrm{C}$. The parameters used in the model are: $d_{o}=28.5 \mu \mathrm{m}, \rho_{l}=$ $1093 \mathrm{kgm}^{-3}, \rho_{\mathrm{s}}=2500 \mathrm{kgm}^{-3}, \lambda=155 \mathrm{kJkg}^{-1}, \alpha=5.7 \times 10^{-7} \mathrm{~m}^{2} \mathrm{~s}^{-1}, C_{p}=0.84 \mathrm{kJkg}^{-1} \mathrm{~K}^{-1}$, $T_{m}=44^{\circ} \mathrm{C}, \phi=22.5^{\circ}, \dot{M}_{D}=8 \mathrm{gmin}^{-1}, \varepsilon_{\mathrm{s}}=0.04, M_{s}=0.6 \mathrm{~kg}, \theta=0.012 \mathrm{~m}^{2} \mathrm{~s}^{-2}, \omega=35.0^{\circ}$. 


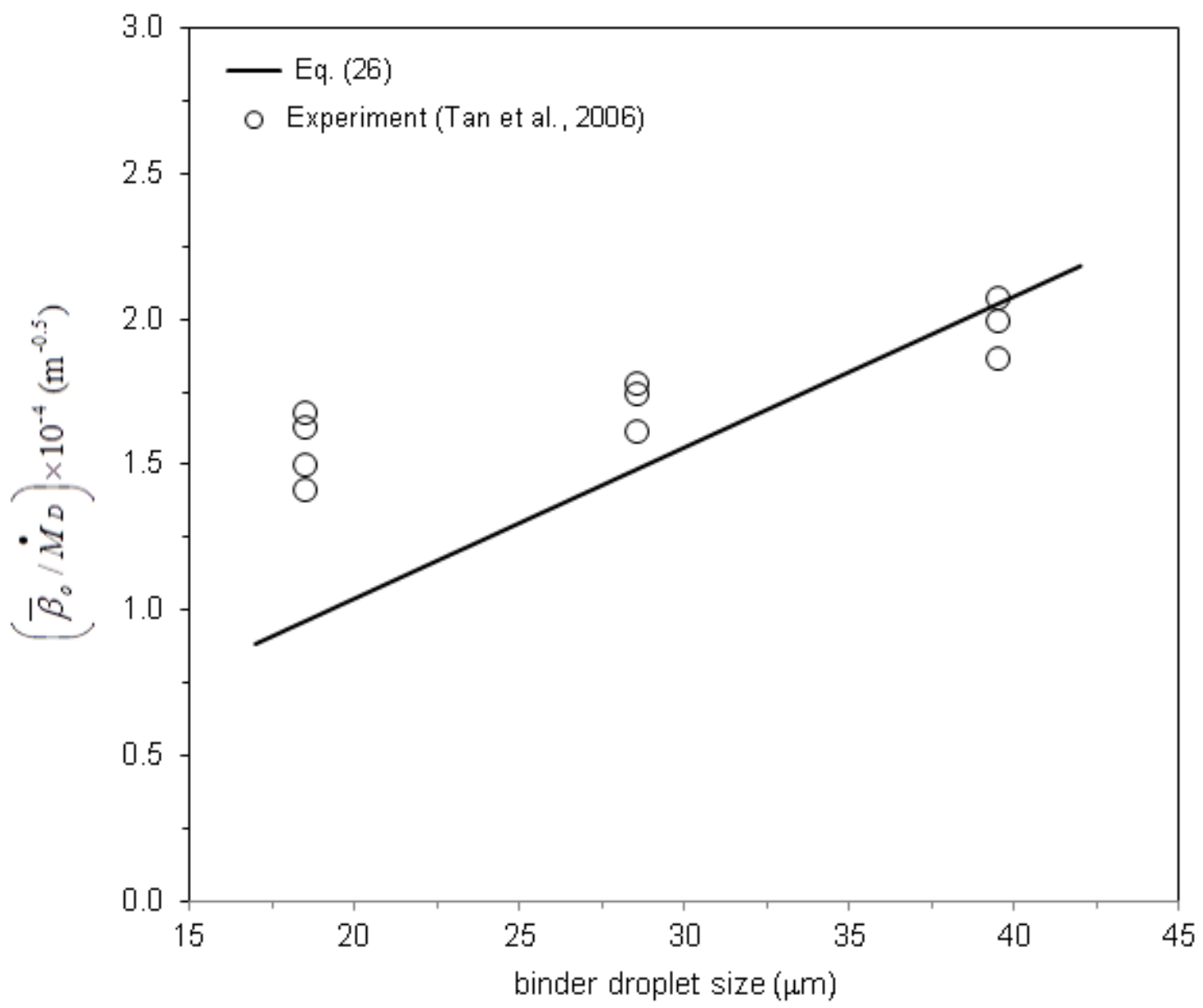

Fig. 1. Comparison of experimental and predicted aggregation rate constant using Eq. (27). The experiment of Tan et al. (2006) was carried out with glass beads of primary size of $175 \mu \mathrm{m}$ and PEG1500 as a binder, fluidizing air velocity of $0.97 \mathrm{~ms}^{-1}$ and a bed temperature of $32{ }^{\circ} \mathrm{C}$. The parameters used in the model are: $D=300 \mu \mathrm{m}, \rho_{l}=1093$ $\mathrm{kgm}^{-3}, \rho_{\mathrm{s}}=2500 \mathrm{kgm}^{-3}, \lambda=155 \mathrm{kJkg}^{-1}, \alpha=5.7 \times 10^{-7} \mathrm{~m}^{2} \mathrm{~s}^{-1}, C_{p}=0.84 \mathrm{kJkg}^{-1} \mathrm{~K}^{-1}, T_{m}=44$ ${ }^{\circ} \mathrm{C}, T_{\mathrm{s}}=32{ }^{\circ} \mathrm{C}, \phi=22.5^{\circ}, \dot{M}_{D}=8 \mathrm{gmin}^{-1}, \varepsilon_{\mathrm{s}}=0.04, M_{\mathrm{s}}=0.6 \mathrm{~kg}, \theta=0.012 \mathrm{~m}^{2} \mathrm{~s}^{-2}, \omega=$ $35.0^{\circ}$ 


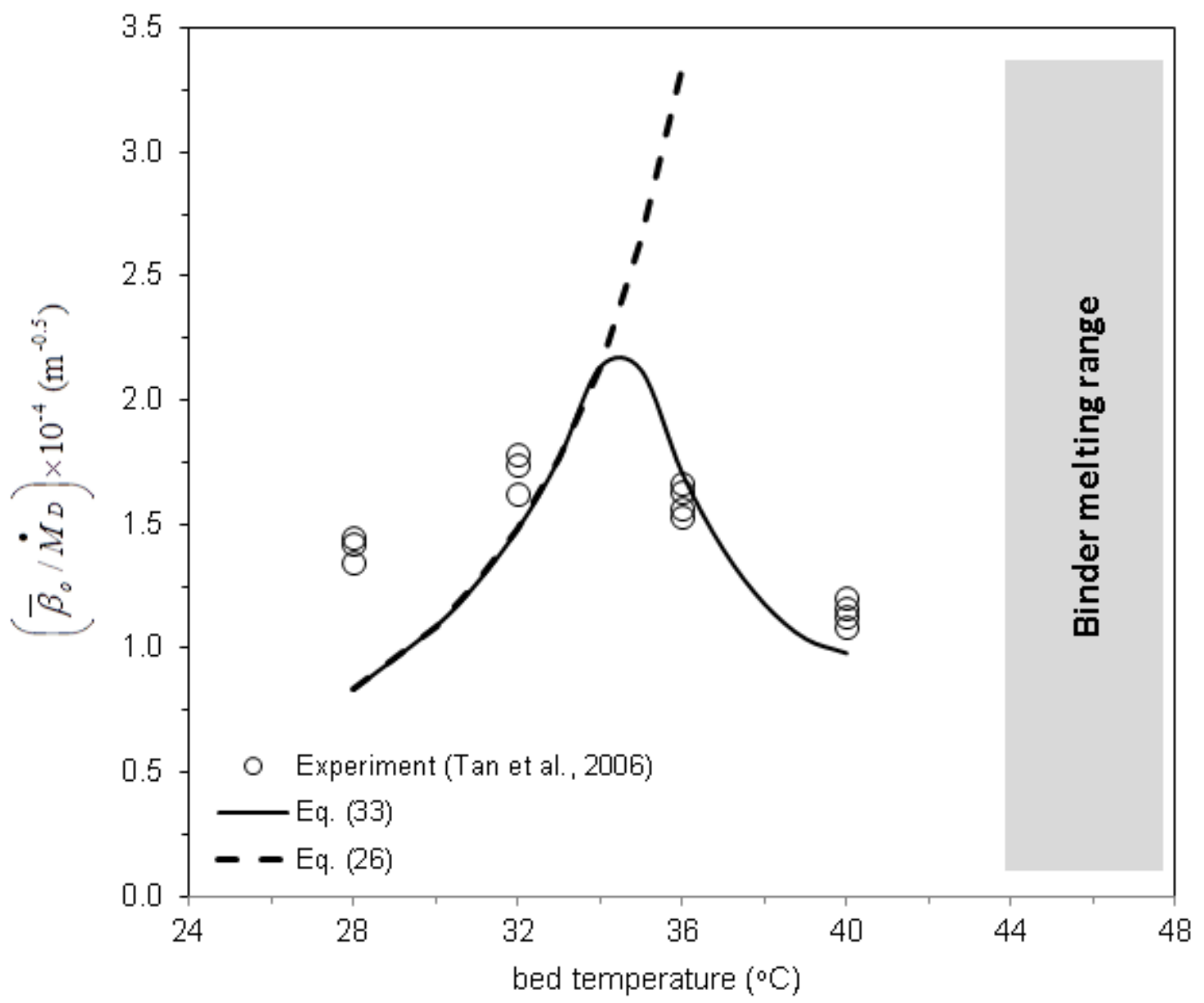

Fig. 7. Comparison of experimental and predicted aggregation rate constant using Eq. (26) and its modified version of Eq. (32). The experiment of Tan et al. (2006) was carried out with glass beads of primary size of $175 \mu \mathrm{m}$ and PEG1500 as a binder, spray nozzle pressure of 1.5 bar and fluidizing air velocity of $0.97 \mathrm{~ms}^{-1}$. The parameters used in the model are: $d_{o}=20 \mu \mathrm{m}, D=300 \mu \mathrm{m}, \rho_{l}=1093 \mathrm{kgm}^{-3}, \rho_{s}=2500 \mathrm{kgm}^{-3}, \lambda=155 \mathrm{kJkg}^{-1}$, $\alpha=5.7 \times 10^{-7} \mathrm{~m}^{2} \mathrm{~s}^{-1}, C_{p}=0.84 \mathrm{kJkg}^{-1} \mathrm{~K}^{-1}, \dot{M}_{D}=8 \mathrm{gmin}^{-1}, T_{m}=44{ }^{\circ} \mathrm{C}, \phi=22.5^{\circ}, \theta=0.012$ $\mathrm{m}^{2} \mathrm{~s}^{-2}, \omega=35.0^{\circ}, \eta=0.95$. 